\title{
Deformation propagation in responsive polymer network films
}

\author{
Surya K. Ghosh, ${ }^{1}$ Andrey G. Cherstvy, ${ }^{1}$ and Ralf Metzler ${ }^{1,2}$ \\ ${ }^{1}$ Institute for Physics 8 Astronomy, University of Potsdam, 14476 Potsdam-Golm, Germany \\ ${ }^{2}$ Department of Physics, Tampere University of Technology, 33101 Tampere, Finland
}

(Dated: 1st March 2018)

\begin{abstract}
We study the elastic deformations in a cross-linked polymer network film triggered by the binding of submicron particles with a sticky surface, mimicking the interactions of viral pathogens with thin films of stimulus-responsive polymeric materials such as hydrogels. From extensive Langevin Dynamics simulations we quantify how far the network deformations propagate depending on the elasticity parameters of the network and the adhesion strength of the particles. We examine the dynamics of the collective area shrinkage of the network and obtain some simple relations for the associated characteristic decay lengths. A detailed analysis elucidates how the elastic energy of the network is distributed between stretching and compression modes in response to the particle binding. We also examine the force-distance curves of the repulsion or attraction interactions for a pair of sticky particles in the polymer network film as a function of the particle-particle separation. The results of this computational study provide new insight into collective phenomena in soft polymer network films and may, in particular, be applied to applications for visual detection of pathogens such as viruses via a macroscopic response of thin films of cross-linked hydrogels.
\end{abstract}

\section{INTRODUCTION}

Cross-linked networks of polymers of varying stiffness are a ubiquitous constituent of biological cells, their plasma membranes, as well as the tissues they make up, and biofilms. Such intra- and extracellular networks include, inter alia, filaments such as microtubules, actin, collagen, fibrin, polysaccharides, or the spectrin network maintaining the shape of red blood cells 1 . In the laboratory, cross-linked polymer gels are widely used for the separation of molecules in setups such as gel electrophoresis [2]. Collective deformations of such elastic networks occur, for instance, due to the motion of individual cells in biofilms or the extracellular matrix, or when large objects such as viruses are taken up and transported inside cells by molecular motors [3].

Elastic deformations and propagation of strain in networks of flexible, semi-flexible, and stiff polymers were analysed in a number of analytical and simulations studies [4 7]. Specifically, both affine and non-affine deformations were studied [8], and the nonlinear stress-strain behaviour investigated [9]. For pre-stressed elastic networks, the rheological properties and the local mechanical response were investigated [10]. Could one use the deformation characteristics of polymeric networks to diagnose the presence of submicron particles with specific surface properties, such as viruses, in the ambient liquid?

Indeed, there exist a number of experimental techniques implementing polymeric networks for the detection of pathogens, including setups based on highly specific aptamers [1] or antibody-antigen interactions [12, 13]. Many of these techniques adapt techniques based on virus immobilisation on supported surfaces. Another strategy for pathogen detection was recently proposed based on experimental studies of polymeric hydrogels [14], and virus detection based on pre-stretched DNA bundles embedded in a hydrogel was rationalised theoretically [15]. Hydrogel-based polymeric and polyelectrolyte materials are known to exhibit a highly responsive behaviour with respect to various external stimuli. The list includes the response to the ambient temperature (including polymeric micro- and nanogels [16, 17]), the solvent quality, $\mathrm{pH}$ value, as well as the presence of various small molecules [18 22]. The shrinkage of polyelectrolyte microgels at elevated temperatures as triggered by temperature-dependent (hydrophobic) interactions was recently studied by simulations [23].

However, volume-based methods of pathogen detection involving hydrogel setups are not expected to have high yields. This is due to the fact that the typical mesh size of the gel network in many cases is comparable to or even smaller than the size of the pathogens (even small viruses measure some 15 to $20 \mathrm{~nm}$ ). The diffusion of such comparatively large objects into the bulk of a hydrogel is strongly impeded and may even exhibit transient anomalous diffusion [24], and these larger particles are thus likely to predominantly occupy a small surface layer. One alternative is to chemically disintegrate the viral components into nucleic acids and capsid proteins or to release easily detectable mobile compounds from the surface of adsorbed particles that then diffuse into the bulk of a biosensor 25] which may be combined with fluorescence-based methods [26]. Another alternative is to use a relatively thin, pseudo two-dimensional supported film of an elastic polymeric material, to which pathogens of varying sizes and adhesive strengths bind [14]. The physical response of this second kind of systems is the main focus of the current study.

Based on extensive Langevin Dynamics simulations we study in detail how elastic deformations of a crosslinked polymer network are effected by introduction of submicron particles into a sticky surface. In particular, we quantify how the deformations propagate into the network, as function of the system parameters given by the network elasticity and the adhesion strength of the particles. The analysed quantities are the collective net- 

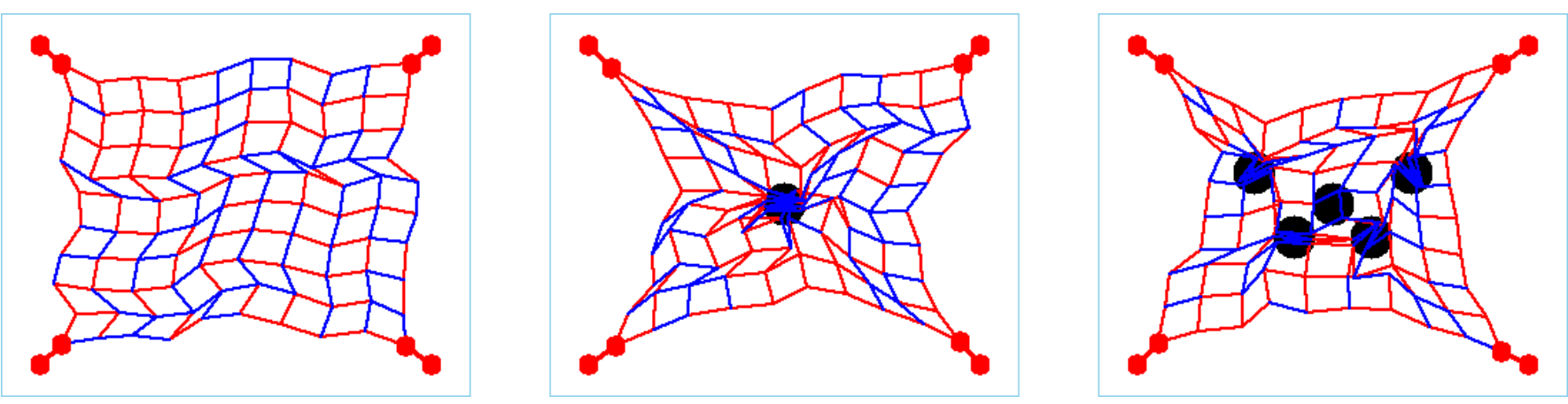

Figure 1: Schematic of the responsive polymeric film supported by fixed linkers at its four corners. Network deformations spontaneously occur due to thermal fluctuations (left) and are enhanced in the presence of a single (middle) or several (right) sticky particles. Stretched bonds are shown in red, while compressed bonds appear blue. A relatively small lattice with an edge length of $n=9$ bonds is shown to illustrate the stretching-compression features. The Supplementary Material contains a video illustrating the dynamics of the film shrinkage for 0,1 , and 5 sticky particles in a relatively soft network and high attraction strength. We use the same parameters for the snapshots shown here: attraction strength $\epsilon_{A}=45 k_{B} T$ and network elasticity $k=15$, see text. The video illustrates that the area initially shrinks rapidly, while at later times it reaches a saturation.

work shrinkage and the associated decay length. We also examine the distribution of the elastic energy in the network, in particular, its partitioning into stretching and compression modes. In addition to effects caused by a single sticky particle we also study the network-mediated interactions of two sticky particles.

In section [I] we specify our simulations and define the quantities to be analysed. In section [II] we present the main results of the simulations and support them with scaling relations. Specifically, we first analyse the binding of a single sticky particle to the thermally activated bead-spring lattice (section IIIA). Section IIIB focuses on static properties and determines the partition of the system, followed by results for the elastic propagation dynamics in section [IIC] The effects for the networkmediated interactions between two particles in the film are examined in section IIID, Finally, we draw our conclusions in section IV.

\section{MODEL}

We use Langevin Dynamics simulations to study the elastic deformations of the two dimensional, discretised lattice-based model of the gel film. The latter consists of a square lattice of Lennard-Jones beads connected with elastic springs, see Fig. 1. The elastic network with a lateral size $n$ (i.e., along one edge we put $n$ bonds) contains $(n+1)^{2}$ beads and $2 n(n+1)$ springs connecting them. We choose the location of the origin in the lattice centre, and for the lattice size $n$ the $x$ and $y$ coordinates then vary from $-\frac{n}{2} \times 2 a$ to $\frac{n}{2} \times 2 a$ with a step size $2 a$. In the simulations we vary the system size in the range $9<n<25$, in order to eliminate boundary effects. Each bead is subjected to the thermal bath, whose noise strength is linked to the temperature of the system. The entire elastic film is anchored at its four corners to maintain the shape and to prevent the total collapse of the network onto the attractive particles, which are treated as immobile. During the simulations we keep track of the elastic energy of the anchoring bonds, which is added to the total elastic energy discussed below.

One of the central quantities we target in our study is the cumulative binding energy $E_{A}$ between a circular sticky particle and the beads of the elastic network. This quantity then provides a basis to gauge the interactions of viral particles with chemically functionalised cross-link points in the polymeric hydrogel network. The strength of the particle-bead adhesion $\epsilon_{A}$ is a model parameter, that can be tuned in experiments via, for instance, a chemical functionalisation of the virus surface changing its affinity to a given polymeric film. It is also sensitive to the solutions conditions, in particular, the concentration and valency of the ambient salt.

The interaction of one of the sticky particles with the beads of the network follows a truncated short-range 6-12 Lennard-Jones (LJ) potential of the form

$$
E_{\text {attr }}(r)=4 \epsilon_{A}\left[\left(\frac{\sigma}{r}\right)^{12}-\left(\frac{\sigma}{r}\right)^{6}\right]+\epsilon_{A}, \text { for } r<2^{1 / 6} \sigma,
$$

and $E_{\text {attr }}(r)=0$ otherwise. The interaction radius of this attractive potential is $r_{a} \approx 0.2 a$, recalling that the lattice constant is $2 a$. This radius controls the number of beads $N_{b}$ counted as bound to the sticky particle in the simulations. Depending of the position of the bead in this attractive potential shell surrounding the sticky particle, the energy gain due to bead adsorption can be smaller than the maximum depth of the attractive potential determined by $\epsilon_{A}>0$. Namely, the magnitude of the overall adsorption energy $E_{A}$ for $N_{b}$ bound beads is then

$$
E_{A} \leqslant N_{b} \epsilon_{A}
$$

The interactions between the network beads are represented by the standard Weeks-Chandler-Andersen repulsive 

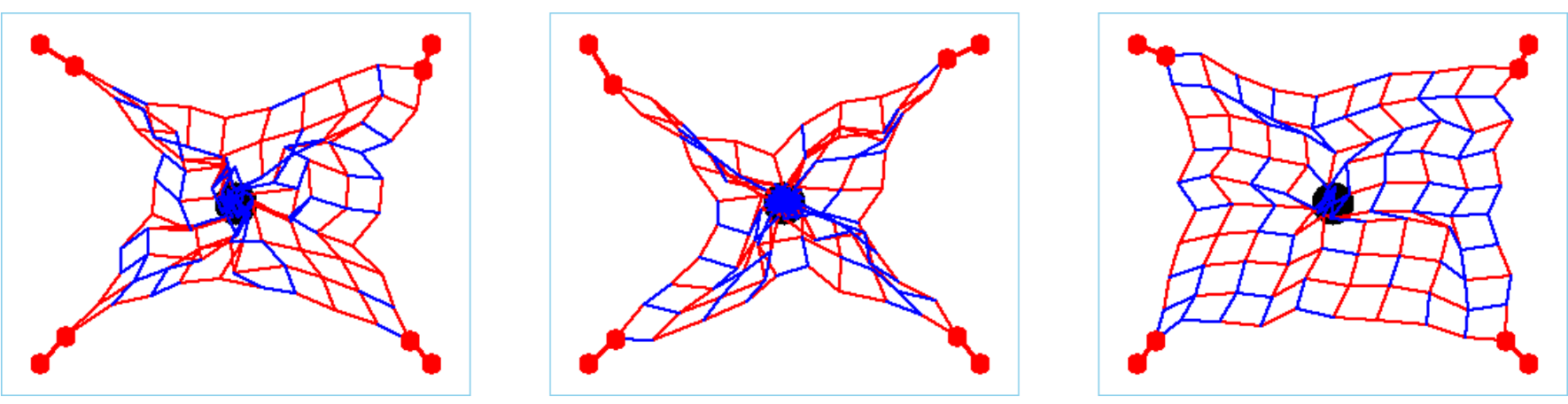

Figure 2: Network response to a sticky particle analogously to Fig. 1. for different bead-particle attraction strengths $\epsilon_{A}$ and network stiffness $k$. Left: $\epsilon_{A}=15$ and $k=15$. Middle: $\epsilon_{A}=45$ and $k=15$. Right: $\epsilon_{A}=15$ and $k=45$.

\section{LJ-like potential [27].}

The network dynamics are governed by the standard Langevin equation in the presence of white Gaussian noise, that independently agitates each bead of the network. If we label each network bead by the numbers $i$ and $j$ denoting its position in the network in $x$ and $y$ direction and call $\mathbf{r}_{i, j}(t)$ this bead's position in the embedding scape, the dynamic equation reads

$$
\begin{aligned}
m \frac{d^{2} \mathbf{r}_{i, j}(t)}{d t^{2}}= & -\sum_{J} \nabla E_{\mathrm{attr}}\left(\left|\mathbf{r}_{i, j}-\mathbf{R}_{v, J}\right|\right) \\
& -\sum_{m, n=1, m \neq i, n \neq j}^{(n+1)} \nabla E_{\mathrm{LJ}}\left(\left|\mathbf{r}_{i, j}-\mathbf{r}_{m, n}\right|\right) \\
& -\nabla \epsilon_{e l,) i, j)}-\xi \mathbf{v}_{i, j}(t)+\mathbf{F}(t)
\end{aligned}
$$

Here $m$ is the mass of the bead, $\xi$ is the friction coefficient, $v_{i, j}$ is the bead velocity, $\mathbf{R}_{v, J}$ is the position of the surface of the sticky particle $J$, and $\mathbf{F}(t)$ represents the Gaussian $\delta$-correlated noise with norm $\left\langle F(t) F\left(t^{\prime}\right)\right\rangle=$ $4 k_{B} T \xi \delta\left(t-t^{\prime}\right)$.

The elastic energy stored in the springs connecting the beads at the bead-bead separation $\delta r_{(i, j)(i+1, j)}=\mid \mathbf{r}_{i, j}$ $\mathbf{r}_{i+1, j} \mid$ is parameterised by the harmonic spring potential with the equilibrium distance $2 a$,

$$
\begin{aligned}
\epsilon_{e l,(i, j)}= & \frac{k}{2}\left[\left(\delta r_{(i, j)(i+1, j)}-2 a\right)^{2}\right. \\
& +\left(\delta r_{(i, j)(i-1, j)}-2 a\right)^{2} \\
& +\left(\delta r_{(i, j)(i, j+1)}-2 a\right)^{2} \\
& \left.+\left(\delta r_{(i, j)(i, j-1)}-2 a\right)^{2}\right] .
\end{aligned}
$$

The elastic constant $k$ varies in our simulations is the range $5 \leqslant k \leqslant 135$ (in dimensionless units), and the total elastic energy of the network is then

$$
E_{e l}=\frac{1}{2} \sum_{i, j=1}^{(n+1)} \epsilon_{e l,(i, j)} .
$$

Depending on the sign of the terms $\delta r_{(i, j)(i+1, j)}-2 a$, we distinguish stretching and compression modes.

For a typical hydrogel the measured Young's modulus $Y$ varies in a broad range $Y=0.01 \ldots 20 \mathrm{kPa}[28$, depending on a number of parameters such as the mesh size and the volume density of the network. The region of dimensionless elastic constants $k$ in our simulations which corresponds to the Young modulus of hydrogels with the mesh size of $50 \mathrm{~nm}$ accommodating typical-size virions is $635<k<5 / 16$. The range of $k$ in our simulations varies within this physical range for hydrogels. Depending on the value of $k$ with respect to the adsorption energy $\epsilon_{A}$, the binding of a sticky particle yields different degrees of film deformation, compare the three snapshots presented in Fig. 2. At every time step we measure the excess elastic energy, that is, the energy on top of the basal energy level for film deformations caused solely by thermal fluctuations, $E_{e l}(t)-E_{e l}^{t h}(t)$. There exist several analogies to our computational model. For instance, the response of elastic gels and the area reduction composed of magnetic particles interconnected by a polymeric network has recently been examined by computer simulations in Ref. 29]. The range of elastic constants and LJ strengths used in that study is similar to our parameter range. We implement the simplest cross-linking method of network beads on a square lattice mimicking the often complex interconnected structures of real hydrogels, see Fig. 7 of Ref. [30].

Our simulations are based on the velocity Verlet algorithm [31] with integration time-step $\Delta t=0.001$. One elementary step in simulation units is converted to $\delta \tau=a \sqrt{m /\left(k_{B} T\right)}$ in real time units. Using realistic parameters for the mesh size of $50 \mathrm{~nm}$ in typical hydrogels [32, 33], we estimate this time as $\delta \tau \approx 6$ ns. In all plots illustrating the dynamics of the system we use the units of simulations time, $\Delta t$. The radius of the sticky particles placed in this lattice is $R=0.9 a$, which is a fixed quantity throughout this study. Unless indicated otherwise, the figures presented below are obtained from averaging over $M=500$ independent realisations of the particle-network system. 

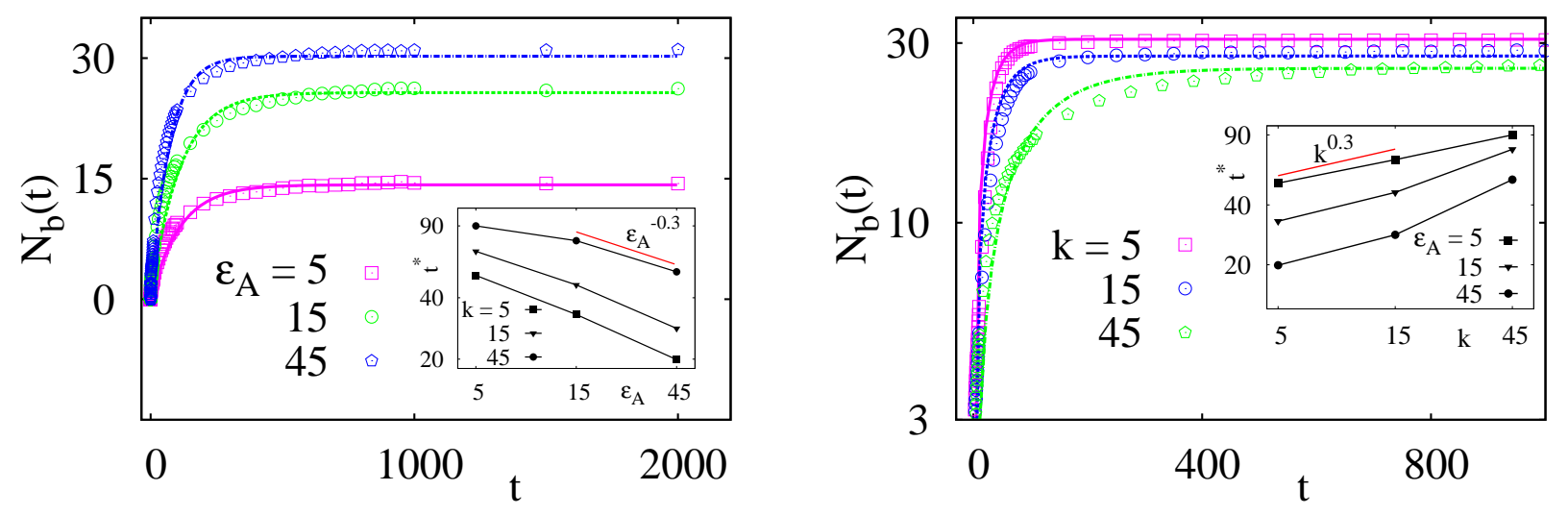

Figure 3: Left: Time dependence of the number $N_{b}(t)$ of bound beads for different attraction strengths $\epsilon_{A}$, for fixed elastic constant $k=45$ and lattice size $n=11$. Fits to Eq. (6) are represented by the lines, and time units are measured in number of simulations steps. $M=500$ realisations are used for averaging. Inset: crossover time $t^{\star}\left(\epsilon_{A}\right)$ from Eq. (6) as function of $\epsilon_{A}$. Right: $N_{b}(t)$ on double-logarithmic scale, showing the difference in convergence to the stationary value $N_{b}^{\max }=\lim _{t \rightarrow \infty} N_{b}(t)$, shown for varying gel stiffness $k$ and for fixed $\epsilon_{A}=15$. Inset: crossover time $t^{\star}(k)$ as function of $k$.

\section{RESULTS}

We present the results of our simulations, starting with the the dynamics of a sticky particle in the network, followed by the the static partition. We then move to the dynamic and static properties when two sticky particles are introduced into the network.

\section{A. Dynamics: number of adsorbed beads, total film area, and elastic energy}

The binding of sticky particles to the adhesive network beads alters the spectrum of their thermal fluctuations. The strength of the particle adhesion governs the interplay between the enthalpic and entropic contributions in the network free energy. Namely, stronger particle binding restricts more severely the conformational freedom of network elements. We observe that softer networks and stronger binding energies of particles trigger more extensive stress propagation in the networks, as expected. Note that the outer contour of the deformed polymer networks observed in the simulations (Fig. 1) remind typical conformations of substrate adhering biological cells 34.

Our simulations show that the number $N_{b}$ of gel lattice beads initially increases quickly with the simulation time $t$ and then saturates to the steady state value $N_{b}^{\max }=\lim _{t \rightarrow \infty} N_{b}(t)$, as shown in Fig. 3] The number of bound beads $N_{b}$ is determined according to the following criterion: if the average bead-particle distance during the simulations is smaller than $R+r_{a}=R+0.2 a$, it is considered as bound. Stronger adhesion strengths $\epsilon_{A}$ trigger a faster initial increase in $N_{b}(t)$, and naturally the plateau value $N_{b}^{\max }$ also increases.

The initial growth of the number of bound beads can be described in terms of a single exponential function according to

$$
N_{b}(t)=N_{b}^{\max }\left(1-e^{-t / t^{\star}}\right),
$$

with the characteristic crossover time $t^{\star}$. As demonstrated in Fig. 3 on the left the quality of the fit in the linear representation is excellent. We observe that the decay time $t^{\star}$ decreases for increasing attraction strengths $\epsilon_{A}$ and decreasing elastic constants $k$. The characteristic time $t^{\star}$ approximately follows the scaling laws

$$
t^{\star}\left(\epsilon_{A}\right) \sim \epsilon_{A}^{-1 / 3}
$$

as function of the attraction strength $\epsilon_{A}$ and

$$
t^{\star}(k) \sim k^{1 / 3}
$$

in dependence of the stiffness constant $k$, compare the insets in Fig. 3. These scaling exponents remain almost constant with varying network size (not shown).

The particle-bead attraction energy $E_{A}$ in the longtime limit is naturally larger than the energy of the elastic deformations of the bonds mediated by the particle binding, as shown in Fig. 4. The thermally-driven network beads are thermally agitated and jiggle around before they get captured by sticky particles, leading to a contraction of adjacent network springs and subsequent compression deformations of further network elements. The network deformation energy is measured as the difference of the entire elastic energy minus the amount of elastic energy caused solely by thermal fluctuations. Namely, in the long-time limit we have

$$
E_{A}>\Delta E_{e l}=E_{e l}-E_{e l}^{t h}
$$

The temporal increase of the adsorption and elastic energies, $E_{A}(t)$ and $E_{e l}(t)$, is a sensitive function of the model parameters. The convergence to the stationary values of the attraction and elastic energies is displayed 


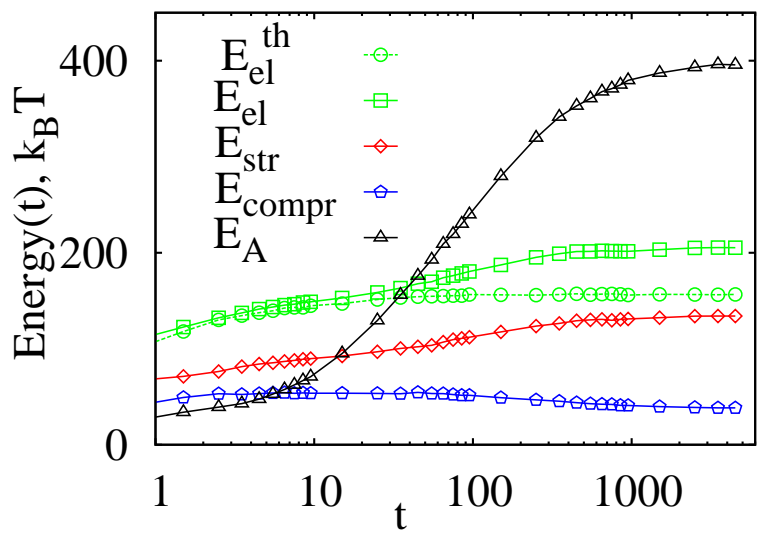

Figure 4: Time dependence of the total attraction energy $E_{A}$ (black) between the network beads and the sticky particle as well as the elastic energy $E_{e l}$ of the bond deformations of the gel (solid green). The latter is the sum of the stretching $E_{\text {str }}(t)$ (red curve) and compression energies $E_{\text {compr }}(t)$ (blue). The network elastic energy solely due to thermal fluctuations, $E_{e l}^{t h}(t)$, is represented by the dashed green curve. The parameters correspond to the situation of Fig. 3. $\epsilon_{A}=15, n=11$, and $k=45$.

in Fig. 4. We also find that larger adsorption strengths $\epsilon_{A}$ and smaller elastic constants $k$ (e.g. $\epsilon_{A}=45, k=$ 15 as contrasted to $\epsilon_{A}=15, k=45$ considered in the majority of plots) cause faster response of the system to particle binding and, as a consequence, faster energy accumulation (not shown). As compared to the thermal elastic energy that scales with the area of the system, $E_{e l}^{t h}(n) \sim(n+1)^{2}$, the elastic deformations mediated by a weakly bound viral particle turn out to be relatively

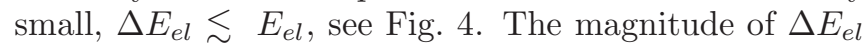
grows when strongly adhesive particles are positioned in the elastic network and the attraction energy exceeds the thermal energy $E_{e l}^{t h}$. Note that relating the attraction energy presented in Fig. 4 to the number of bound beads shown in Fig. 3 we conclude that due to binding to the particle surface not all beads gain the maximum attraction energy given by $\epsilon_{A}$, as indicated in Eq. (2).

Fig. 5 on the left illustrates the changes in the network area $A(t)$ due to adsorption of network beads to the sticky particle. We see that with increasing attraction strength $\epsilon_{A}$ the film area decreases faster and reaches smaller stationary values as compared to a free film in response to thermal fluctuations. We observe that the area shrinkage with time is a sensitive function of the attraction strength $\epsilon_{A}$. The shrinkage dynamics can be fitted by the relaxation function

$$
A(t) / A_{0}=e^{-t / t^{*}}+C\left(1-e^{-t / t^{*}}\right),
$$

with a single exponential, where $A_{0}=(2 a n)^{2}$ is the initial network area, and the parameter $C=C\left(\epsilon_{A}, k\right)$ accounts for different plateau values of $A / A_{0}$ at long times, as function of $\epsilon_{A}$ and $k$. The fit to the simulations data is particularly good when the relative area change in the course of the system is substantial (for large $\epsilon_{A}$ and small $k$ ). To perform a systematic fit for both the number of bound beads in Fig. 3 and the area of the network in Fig. (5) We considered the time span during which $90 \%$ of the area shrinkage is completed.

The number of bound beads $N_{b}(t)$ reflects the local binding characteristics and naturally grows with the strength of the bead-particle attraction. In contrast, the area of the film is a global property, which involves also non-affine deformations. The latter contain, for instance, tilts and other rearrangements of rhomb-like network elements, leaving the elastic energy of the network approximately unchanged.

The decay time $t^{*}$ of the initial relative area drop is an increasing function of the attraction strength $\epsilon_{A}$, as

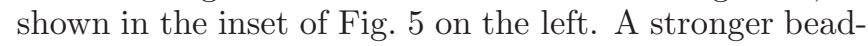
particle attraction strength $\epsilon_{A}$ causes more extensive and longer-ranged deformations of the network, such that also more distant beads attach to the sticky particle surface at later times. This effects a longer time scale $t^{\star}$. Likewise, the response of more elastic networks is faster but the relaxation time $t^{\star}$ slightly grows for smaller $k$ values, see

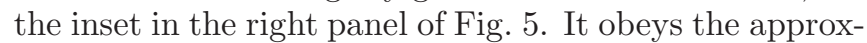
imate scaling relations $t^{*}\left(\epsilon_{A}\right) \sim \epsilon_{A}^{1 / 5}$ and $t^{*}(k) \sim k^{-1 / 5}$. We also observe that this scaling of the decay time varies only marginally with the system size (not shown).

Finally, for varying network stiffness we observe, as expect naively, that softer networks allow much more extensive film deformations, compare the curves in Fig. [5 on the right. The initial area change follows again the exponential law, $A(t) / A_{0} \sim \exp \left(-t / t^{*}\right)$. The inset in Fig. 5 on the right illustrates the dependence of the decay time of area shrinkage on the network elasticity.

\section{B. Steady state: energy partitioning in the gel}

The variation of the cumulative steady state elastic energy of the polymer film is shown in Fig. 6] This total energy is the sum of the stretching $E_{\text {str }}$ and compression $E_{\text {compr }}$ energies, which at long times are written as

$$
E_{e l}(r)=E_{s t r}(r)+E_{\text {compr }}(r) .
$$

As seen from Fig. 6 the total elastic energy contained within a radius $r$ away from the centre of the introduced sticky particle grows quickly at smaller values of $r$ and then saturates. In other words, most of the elastic energy is accumulated in the vicinity of the sticky particle. As function of the attraction strength $\epsilon_{A}$ shows that higher values of $\epsilon_{A}$ cause more distant network deformations in close proximity of the adsorbed particle, see also the middle panel of Fig. 2. To rationalise this effect, we determine the effective radius $R_{a}$ of network deformation around the centre of the sticky particle that contains $90 \%$ of the elastic energy. The dependence of $R_{a}$ on the attraction strength $\epsilon_{A}$ and the network stiffness $k$ is shown in the inset of Fig. 6. Physically this result implied that stronger particle-bead attractions cause more extensive 

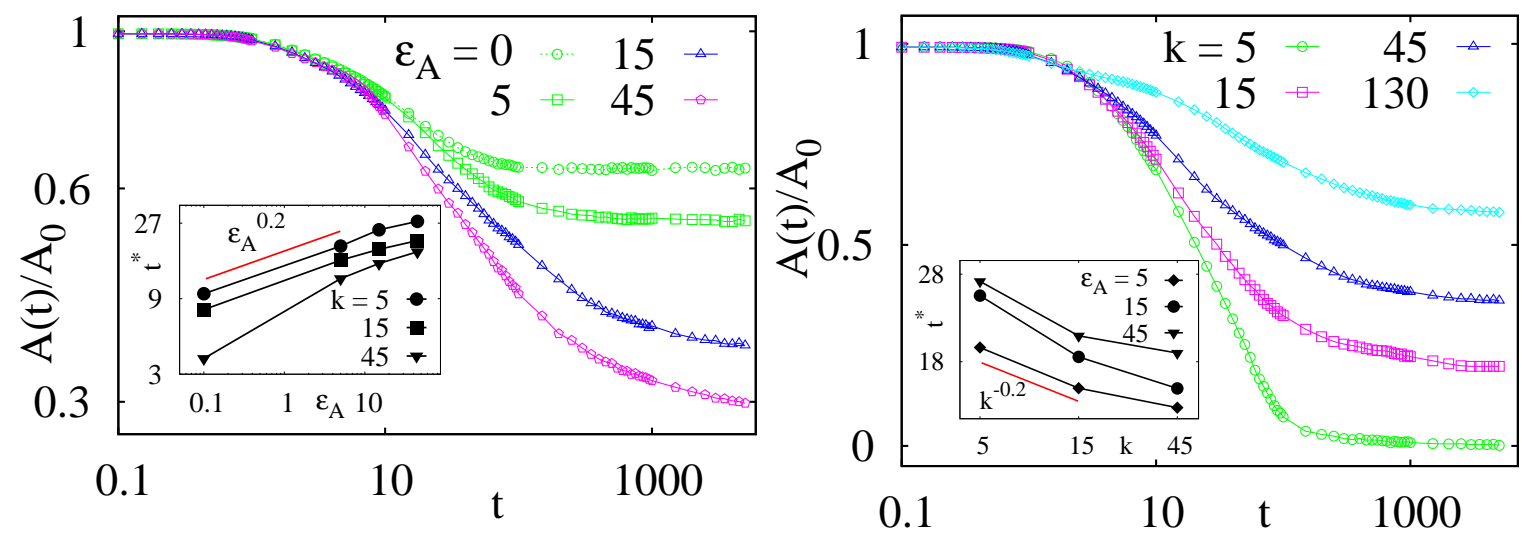

Figure 5: Left: Time dependence of the relative network area $A(t) / A_{0}$ for different attractions strengths $\epsilon_{A}$. The area shrinkage in the absence of sticky particles is the dashed green curve. The inset illustrates the dependence of the crossover time $t^{*}\left(\epsilon_{A}\right)$ in Eq. (10) on $\epsilon_{A}$. The points at $\epsilon_{A}=0$ correspond to the area relaxation of the lattice purely due to thermal effects in absence of sticky particles. The parameters are the same as in Fig. 4 Right: Area shrinkage for varying network stiffness constant $k$. The inset shows the crossover time $t^{*}(k)$.

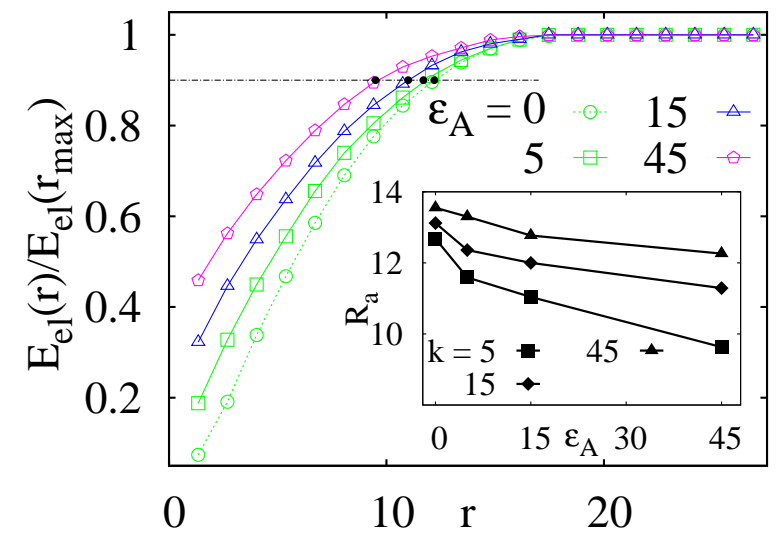

Figure 6: Total elastic energy $E_{e l}(r)$ as function of the relative distance from the sticky particle in the steady state of the lattice deformation. The radii $R_{a}$ of circles containing $90 \%$ of $E_{e l}$ (horizontal black dashed-dotted line in the main graph) are shown in the inset. Energies are normalised to their maximal values achieved at $r=r_{\max }$. Parameters: $n=19, k=15$.

network deformations, while softer elastic networks yield more localised film deformations (smaller $R_{a}$ values for smaller $k$ values), in accordance with intuition.

The stretching and compression components of the elastic energy yield different magnitudes close to the surface of the sticky particle ("core" region) and further away ("bulk" of the film). Specifically, in the vicinity of the core the compression energy dominates, while on the boundary of the elastic film the stretching energy acquires larger values. The profiles of the stretching and compression energies as function of the radial separation $r$ from the centre of the sticky particle in the steady state are shown in Fig. 7 on the left. We observe that at pro- gressively larger separations from the adsorbed particle the energy profiles are nearly flat meaning that the network bulk contribute only little to the deformation energy.

This energy partitioning is partially due to the fact that the entire system is anchored at its four corners (Fig. 1). As the sticky particle is smaller than the lattice spacing $2 a$, the compression of the bonds takes place in the core region to enable binding of separated network beads to the attractive particle surface. Accompanying such a - generally non-affine - core compression, the boundary of the system rather reveals stretching deformations, as witnessed in Fig. 7 The the anchoring points and the lattice boundary effects also impact the interaction energy of two particles in the network, see section

Fig. 7 on the right illustrates the elastic energy partitioning as computed in a layer-by-layer construction on the non-deformed lattice up to the maximum radius $r_{\max }=2 \sqrt{2} \frac{n}{2} a$. We observe that for the bare, thermally agitated elastic film the stretching and compression energies in the central region are equally distributed. At the film boundary the entire elastic energy is due to stretching of the lattice bonds, compare Fig. 1. In contrast, when a strongly attractive particle is introduced, its surface compresses the network, and the disproportions of the stretching and compression energies in the core region of the film (small $s$ values) become apparent.

\section{Dynamics: network deformations around two viral particles}

We now turn to study the effects of two sticky particles in the elastic network, in particular, the particle-particle 

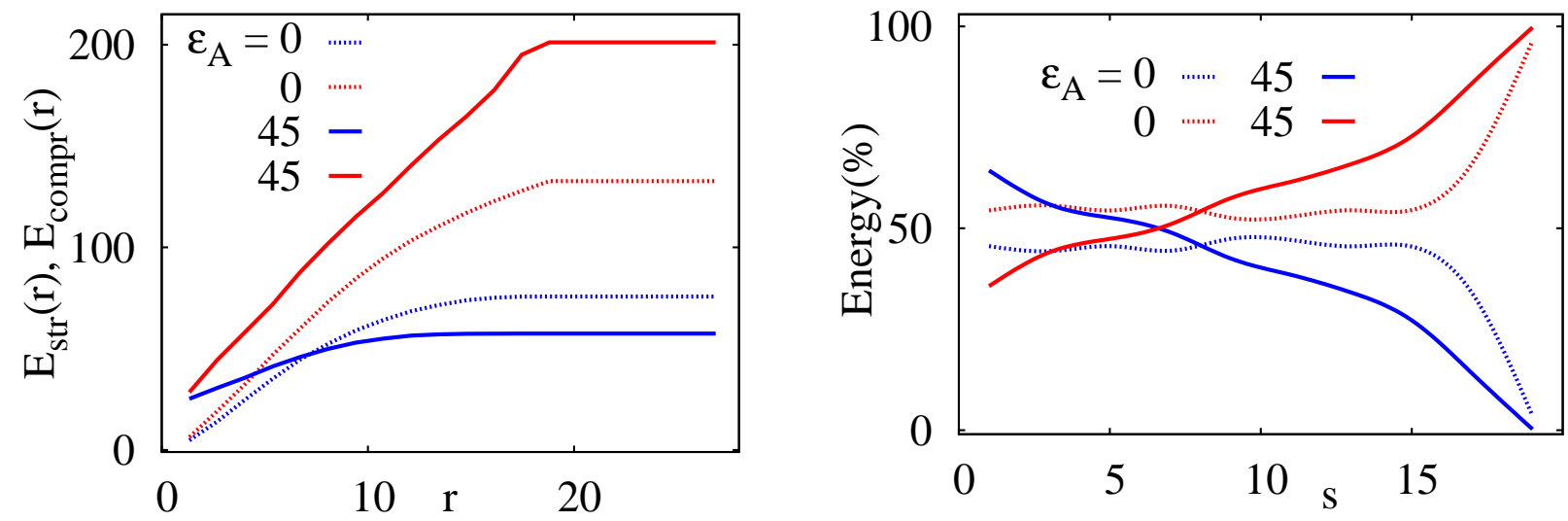

Figure 7: Left: steady state distribution of stretching $\left(E_{s t r}(r)\right.$, red $)$ and compression $\left(E_{c o m p r}(r)\right.$, blue) energies of the network away from the adsorbed particle. The physical distance $r$ is the separation from the particle in the deformed lattice, with $r_{\max }=2 \sqrt{2} \frac{n}{2} a$. For comparison, the energy profiles in the absence of bound particle $\epsilon_{A}=0$ are shown as well. Parameters: $k=45, n=19$. Right: Relative stretching (red) and compression (blue) energies as functions of the lattice site number $1<s<n$ for the non-deformed film. For comparison, the energy profile of the bare network is shown as the dotted lines. The sum of the corresponding red and blue curves at each $s$ always amounts to unity.
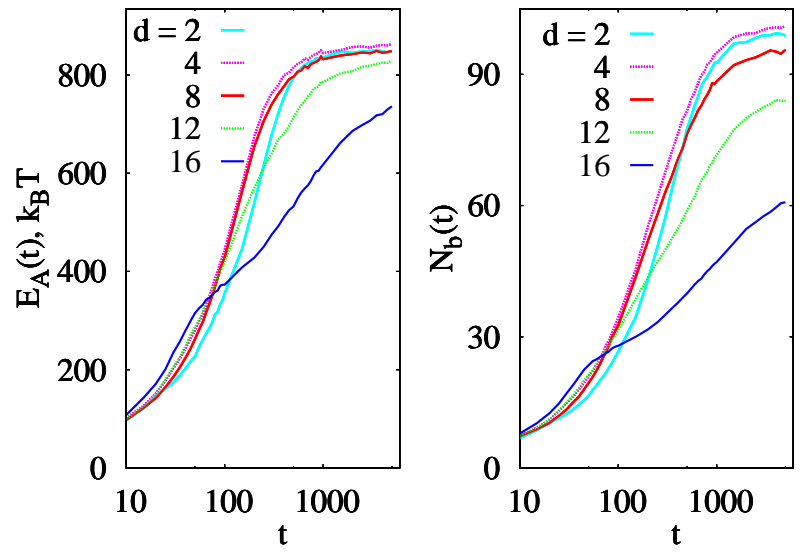

Figure 8: Time dependence of the number $\mathcal{N}_{b}(t)$ of bound network beads and the total adhesion energy $\mathcal{E}_{A}(t)$ of beads to two viral particles introduced into the network. The particles are separated by the distance $d /(2 a)$, as indicated in the plots. Parameters: $n=25, k=45$, and $\epsilon_{A}=15$.

interactions. Varying the centre-to-centre separation $d$ between these particles, we enumerate how the number $\mathcal{N}_{b}(t)$ of bound network beads increases with time, as shown in Fig. 8 (here and below curly letters denote twoparticle quantities). We find that for large inter-particle separations $d$ the number of bound beads is considerably smaller than for closely-positioned particles, indicating boundary effects.

As compared to a single sticky particle introduced into the same network, the number of bound beads increases approximately twice as fast, compare Fig. 9. At long times, two particles are somewhat less efficient in deforming the network compared to twice the single particle effect. Thus, some frustration and mutual impediment of the action of two particles occurs.
A reason for the fact that the number of bound beads and the adsorption energy are not simply proportional to one another goes as follow. According to the adsorption criterion we defined, beads within the attraction radius are counted as adsorbed (their favourable attraction energy is not yet in the minimum of the potential). In the course of adsorption, the beads rearrange and their attraction energy increases in magnitude. At relatively large separations between the sticky particles, the anchoring points of the film start to influence the network response, impeding bead adsorption to the sticky particles. This is the likely reason for the apparent kink in the time dependence of the bead adsorption energy $\mathcal{E}_{A}(t)$ at short times for well separated particles as well as for the number $\mathcal{N}_{b}(t)$ of bound beads, see the lowest curves at $d /(2 a)=16$ in Fig. 8 . We also observe that the elastic network energy for the adsorption of two relatively weakly-adhering sticky particles, $\mathcal{E}_{e l}$, is not significantly different from that for a single adsorbed particle (not shown). In a much larger network, obviously the contribution from two well separated sticky particles is necessarily additive. However, for the realistic system we have in mind, the finiteness of our simulations network reflects the attachment of the hydrogel to its solid support in the corners.

We note that in the steady-state we observe a nontrivial dependence of the number $\mathcal{N}_{b}(d)$ of adsorbed beads. Namely, for very closely positioned particles, $d /(2 a) \approx 1 \ldots 3$, the number of bound beads decreases, indicating a shortage of the network material in between the two particles capable of adsorption. For small separations $d$ the beads in between the two sticky particles are frustrated in their tendency to bind to either of the two attractive surfaces. This represents a cause for repulsive particle-particle interactions at very close separations $d$, see below. 


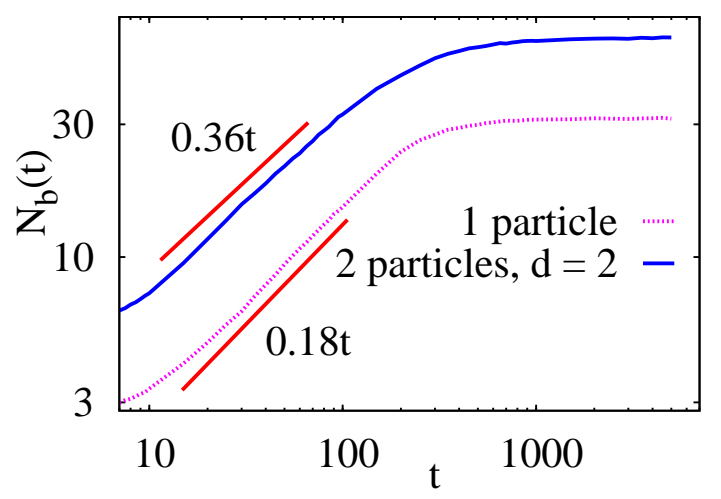

Figure 9: Comparison of the number of bound beads when two sticky particles are in the network to the case of a single particle. Parameters are the same as in Fig. 8

\section{Steady-state: Network-mediated particle-particle interactions}

We finally address the question of the partitioning of the elastic energy in the network in the presence of two sticky particles, in particular, as function of the particleparticle separation $d$.

In this context it is worthwhile remarking that in the context of lipid membranes the topic of membranemediated attractive interactions between spherical colloidal particles [35 37] and cylindrical DNA molecules deposited on cationic lipid membranes [38, 39] attracted significant theoretical and experimental attention in recent years. The reason for inter-particle attraction put forward in some fluctuation-based models is due to the tendency to reduce the inter-particle separation in order to diminish the area of a deformed membrane in which fluctuations are suppressed due to binding of adhesive particles. In curvature-based fluctuation-free models of membrane-mediated attraction, in contrast, the reduction of the membrane area with large curvature gradients in the proximity of adhered particles, that deform the membrane substrate, causes their mutual membranemediated attraction [39].

To quantify the interaction energies $E_{\text {int }}(d)$ we determine the difference of the combined adsorption $\left(\mathcal{E}_{A}(d)\right)$ and elastic $\left(\mathcal{E}_{e l}(d)\right)$ energies for two sticky particles and the same quantities for a single sticky particle in the same lattice $\left(E_{A}\right.$ and $\left.E_{e l}\right)$, that is

$$
E_{\text {int }}(d)=\left[\mathcal{E}_{A}(d)+\mathcal{E}_{e l}(d)\right]-2\left(E_{A}+E_{e l}\right) .
$$

In contrast to the membrane case, in which interactioninduced wrapping of the membrane around adhesive particles may occur and thus the membrane assumes outof-plane deformations triggering the attraction, in our planar film the network deformations are restricted to the $x-y$ plane. According to Eq. (12) at large inter-particle separations $d$ the two particles deform the film to the same extent as twice the effect of a single particle, see also the long time behaviour in Fig. 9 Thus, in ab-
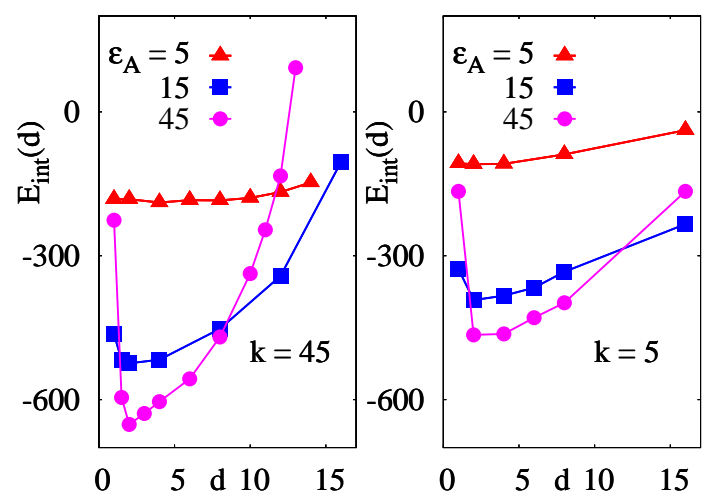

Figure 10: Inter-particle network-mediated interaction energy $E_{\text {int }}(d)$ for different attraction strengths $\epsilon_{A}$ (left panel) and for varying elasticity constants $k$ (right panel). The interaction energy depth grows with $\epsilon_{A}$. A non-zero value of $E_{\text {int }}\left(d=d_{\text {max }}\right)$ for the largest $\epsilon_{A}$ indicates a growing influence of boundary effects. Parameters: $n=25, k=5$.

sence of boundary effects $(n \gg 1)$ one expects the limit $E_{\text {int }}(d \rightarrow \infty)=0$. Note that finite lattice sizes in simulations preclude this convergence. The relative contribution of the adsorption energy versus the film deformation term in Eq. (12) grows with the attraction strength $\epsilon_{A}$.

We observe that the particle-particle interaction energy is non-monotonic in the mutual separation $d$. Interactions are attractive in the region of a positive force, that is, when $f_{\text {int }}(d)=-(\partial / \partial d) E_{\text {int }}(d)<0$, while at close inter-particle distance $d$ the network-mediated interactions are repulsive. At large $d$ the interaction energy increases due to a prohibited stretching of the film boundary. In the analysis we thus should keep in mind this finite-size effect of the film when $d /(2 a)$ becomes comparable to the lattice size $n$. Also note that at high concentrations of sticky particles introduced into the film (right panel in Fig. 1), when the average distances are in the region of attraction we just estimated, these particles aggregate into larger complexes.

The network-mediated particle-particle attraction becomes more pronounced at higher values of the attraction strength $\epsilon_{A}$, compare Fig. 10. The magnitude of the particle-particle attraction, given by the maximum depth of the attraction energy well $E_{\text {int }}^{\max }$, naturally grows for stronger bead-particle attraction $\epsilon_{A}$ and decreases for smaller network elasticity $k$, as shown in Fig.10. A somewhat counter-intuitive feature is the diminished attraction strength for softer networks due to extensive binding of effectively volume-free network elements to the sticky particles. Therefore, the "self-energy" of an individual particle introduced into the network is a relatively large number, with a large portion of network beads already bound to the particle, as one can deduce from Fig. 5 . At the same time the interaction energy, Eq. (12), which is the relative contribution in the particle pair as compared to the contribution of two well separated particles, gets smaller at smaller $k$ values because of an impeded propensity of binding even more network beads to the 
sticky particles, i.e., above-mentioned frustration effect.

\section{DISCUSSION AND OUTLOOK}

We presented a simple conceptual framework to rationalise the elastic response of a two-dimensional elastic film to the binding of sticky particles. From the dynamical point of view, the kinetics of area shrinkage due to progressive binding of network elements was quantified. In particular, we obtained simple scaling relations for the characteristic relaxation times for the number of bound network beads and the film area, as functions of the network stiffness and the attraction strength of the particles to the network beads. In the steady-state, we obtained the distribution of the elastic energy as function of the distance from the adsorbed particle and also quantified the radius of propagation of the elastic deformations. Finally, we enumerated the interaction energies and forces acting between sticky particles in the network which emerge due to the elastic network deformations.

The observed strengthening of the effective particleparticle attraction for stronger bead-particle attraction strength is similar to trends for membrane-mediated attractions between parallel DNA molecules adsorbed on unsupported cationic lipid membranes which wrap around the negatively charged DNAs [39]. An every-day life analogy for this network-mediated attraction between the sticky particles as well as for the distribution of network deformations in the vicinity of a pathogen can in fact be the example of two persons on a trampoline. In fact, this simple analogy is particularly close when the trampoline's fabric is only anchored in its four corners.
We also note that the interactions between sticky particles deposited on soft responsive networks are akin to capillary forces acting between particles immersed on fluid interfaces 40 42]. The inter-particle attraction and clustering emerges as a consequence of the reduction of regions of network deformations with high elastic energy gradients, focused in the proximity of adsorbed particles.

As mentioned in the introduction, the results we obtained here may open new perspectives for the detection of particles such as viral pathogens of different binding specifics by help of thin highly-responsive polymeric films. The current area-shrinkage setup for viral particle detection thus complements the model of detection we proposed recently, which converts the virus-DNA binding events and accompanying DNA melting into measurable volumetric changes of the hydrogel specimen [15]. To exploit the whole phase space for such purposes, future simulations should include particles with asymmetric geometries (ellipsoidal or rod-like shapes, for instance 43]) introduced into a three-dimensional inter-connected network, instead of the simple 2D planar case examined here. In this situation, anisotropic deformations of the network and anisotropic inter-particle interactions will occur. These are the targets for future investigations.

\section{Acknowledgments}

The authors thank J. Shin and A. Laschewsky for discussions. We acknowledge funding from the Academy of Finland (FiDiPro scheme to RM), the German Research Council (DFG Grant CH 707/5-1 to AGC), and the Federal Ministry of Education and Research (SKG).
[1] B. Alberts, D. Bray, J. Lewis, M. Raff, K. Roberts, and J. D. Watson, Molecular Biology of the Cell (Garland Publishing, New York, NY, 1994); R. Phillips, J. Kondev, and J. Theriot, Physical Biology of the Cell (Garland Science, New York, NY, 2009).

[2] D. Sean and G. W. Slater, Electrophoresis 33, 1341 (2012); S. Fisher and L. Lerman, Cell 16, 191 (1979).

[3] G. Seisenberger et al., Science 294, 1929 (2001).

[4] J. Wilhelm and E. Frey, Phys. Rev. Lett. 91, 108103 (2003).

[5] D. A. Head, A. J. Levine, and F. C. MacKintosh, Phys. Rev. Lett. 91, 108102 (2003).

[6] R. Everaers, New J. Phys. 1, 12.1 (1999).

[7] Q. Wen, A. Basu, J. P. Winder, A. Yodh, and P. A. Janmey, New J. Phys. 9, 428 (2007).

[8] M. Rubinstein and S. Panyukov, Macromol. 30, 8036 (1997).

[9] H. Kang, Q. Wen, P. A. Janmey, J. X. Tang, E. Conti, and F. C. MacKintosh, J. Phys. Chem. B 113, 3799 (2009).

[10] D. A. Head and D. Mizuno, Phys. Rev. E 88, 022717 (2013).

[11] R. Wang and Y. Li, Biosens. Bioelectr. 42, 148 (2013).
[12] M. S. Ozdemir et al., Anal. Chem. 85, 4770 (2013).

[13] M. Vestergaard, K. Kerman, and E. Tamiya, Sensors 7, 3458 (2007).

[14] E. Wischerhoff, N. Badi, J. F. Lutz, and A. Laschewsky, Soft Matter 6, 705 (2010).

[15] J. Shin, A. G. Cherstvy, and R. Metzler, arXiv:1310.5531.

[16] G. Aguirre, J. Ramos, and J. Forcada, Soft Matter 9, 261 (2013).

[17] J. Ramos, J. Forcada, and R. Hidalgo-Alvarez, Chem. Rev. 114, 367 (2014).

[18] T. Yang, R. Ji, X.-X. Deng, F.-S. Du, and Z.-C. Li, Soft Matter, at press; DOI:10.1039/C3SM53059K .

[19] R. Nigmatullin, M. Benscik and F. Gao, Soft Matter 10, 2035 (2014).

[20] J. Li, Z. Suo, and J. J. Vlassak, Soft Matter, at press; DOI:10.1039/C3SM52751D.

[21] O. Okay, Prog. Polym. Sci. 25, 711 (2000).

[22] O. Okay, in Hydrogel Sensors and Actuators, edited by G. Gerlach and K.-F. Arndt, Springer Series on Chemical Sensors and Biosensors 6, 1 (2009).

[23] M. Quesada-Perez and A. Martin-Molina, Soft Matter 9, 7086 (2013).

[24] A. Godec and R. Metzler (unpublished). 
[25] S. K. Ghosh, T. Kundu, and A. Sain, Phys. Rev. E 86, 051910 (2012).

[26] S. Inal, J. D. Kölsch, F. Sellrie, J. A. Schenk, E. Wischerhoff, A. Laschewsky, and D. Neher, J. Mater. Chem. B 1, 6373 (2013).

[27] J. D. Weeks, D. Chandler and H. C. Andersen, J. Chem. Phys. 54, 5237 (1971).

[28] M. Ahearne, Y Yang, A. J. El Haj, K. Y. Then, and K. K. Liu, J. Royal Soc. Interface 2, 455 (2005).

[29] R. Weeber, S. Kantorovich, and C. Holm, Soft Matter 8, 9923 (2012).

[30] W. Richtering and B. R. Saunders, Soft Matter, DOI: 10.1039/C4SM00208C.

[31] M. P. Allen and D. J. Tildesley, Computer simulations of liquids (Clarendon Press, Oxford, UK, 1987).

[32] M. Betz, J. Hörmansperger, T. Fuchs, and U. Kulozik, Soft Matter 8, 2477 (2012).

[33] J. L. Yaguea and K. K. Gleason, Soft Matter 8, 2890 (2012).

[34] E. Sackmann and A.-S. Smith, Soft Matter 10, 1644 (2014).
[35] B. J. Reynwar, G. Ilya, V. A. Harmandaris, M. M. Müller, K. Kremer, and M. Deserno, Nature 447, 461 (2007).

[36] B. J. Reynwar and M. Deserno, Soft Matter 7, 8567 (2011), and references therein.

[37] S. Dasgupta, T. Auth, and G. Gompper, Soft Matter 9, 5473 (2013).

[38] D. Harries, S. May, and A. Ben-Shaul, Soft Matter 9, 9268 (2013).

[39] A. G. Cherstvy and E. P. Petrov, Phys. Chem. Chem. Phys. 16, 2020 (2014).

[40] K. D. Danov and P. A. Kralchevsky, Adv. Coll. Interf. Sci. 154, 91 (2010).

[41] E. P. Lewandowski, J. A. Bernate, A. Tseng, P. C. Searson, and K. J. Stebe, Soft Matter 5, 886 (2009).

[42] H. Rezvantalaba and S. Shojaei-Zadeh, Soft Matter 9, 3640 (2013).

[43] S. Dasgupta, T. Auth, and G. Gompper, Nano Lett. 14, 687 (2014). 